\title{
Influence of DNA Methylation and Core Binding Factors in Expression of Leukemia Virus in Hemapoietic Stem Cells
}

\author{
Manoj G. Tyagi ${ }^{1 \star}$ and Aniket Kumar ${ }^{1}$ \\ ${ }^{1}$ Department of Pharmacology, Christian Medical College, Vellore 632002,
} Tamil Nadu, India.

Review Article

Received $8^{\text {th }}$ July 2011

Accepted $13^{\text {th }}$ July 2011

Online Ready $18^{\text {th }}$ July 2011

\section{ABSTRACT}

Retroviral vectors based upon the Moloney murine leukemia virus (MLV) have been used due to their high efficiency of stable gene transfer. Core binding factors (CBF) are heterodimeric transcription factors containing a DNA binding Runx 1, Runx 2, or Runx 3 subunit, along with a non DNA binding CBF $\beta$ subunit. All four subunits are required at one or more stages of hematopoiesis. This review describes the role of Runx 1 and CBF $\beta$ in the initiation of hematopoiesis in the embryo, and in the emergence of hematopoietic stem cells. The core site in the Moloney murine leukemia virus (Moloney MLV) enhancer was previously shown to be an important determinant of the T-cell disease specificity of the virus. Mutation of the core site resulted in a significant shift in disease specificity of the Moloney virus from T-cell leukemia to erythro-leukemia. It has been shown that a protein that binds the core site, one of the core-binding factors is highly expressed in thymus and is essential for hematopoiesis in stem cells. Earlier studies suggest that CBF plays a critical role in mediating pathogenesis of Moloney MLV in vivo. Spontaneous leukemia was not observed either upon CBF expression, consistent with a model in which the increase in HSC and progenitor populations represents a pre-leukemic state, and additional mutations are required for progression to leukemia.

Keywords: Leukemia; core binding factor; stem cell; methylation; hematopoiesis; 


\section{INTRODUCTION}

Transcriptional repression is thought to be mediated by both cis-acting de novo methylation of the integrated pro-viruses and cell type specific transcripting transcriptional repressors. The effect of trans-acting factors on retroviral expression through binding of specific sequences within the promoters of retroviruses has been examined in many studies (Ganstch, 1980). In context, the role of methylation in silencing has been less clear. DNA methylation is thought to be a general mechanism used by cells to silence foreign DNA and may be involved in the cell defense against transposable elements. DNA methylation has also been associated with the repression of gene expression and the silencing of viral control elements (Cedar, 1998, Hoeben et al., 1991, Ivanov, 2011). On the other hand affinity of CBF for most core sites found in MLV enhancers, introduced sites with different affinities for CBF into the Moloney MLV genome, has determined the effects of these sites on viral pathogenesis. A correlation between CBF affinity and the latent period of disease onset, in that Moloney MLVs with high-affinity CBF binding sites induced leukemia following a shorter latent period than viruses with lower-affinity sites.

\section{RECENT ADVANCES IN THE REGULATION OF LEUKEMIA VIRUS EXPRESSION}

Numerous studies have underscored the importance of CBF in hematopoiesis. CBF is a heterodimer consisting of a DNA binding subunit called CBFa and a non-DNA-binding subunit, CBFb (Loh et al., 1990; Yoder et al., 1997). Three related genes encode CBFa subunits (Cbfa1, Cbfa2, and Cbfa3), and one gene encodes the common CBFb subunit (Cbfb) (Kundu et al., 2002; Sasaki et al., 1996). Homozygous disruption of two of the CBF genes in mice, Cbfa2 and Cbfb, blocks fetal liver hematopoiesis (Niki et al., 1997; Wang et al., 1996). All definitive hematopoietic lineages are affected by the Cbfa2 and Cbfb mutations, suggesting that $\mathrm{Cbfa} 2$ and $\mathrm{Cbfb}$ are required at the level of the pluripotent hematopoietic stem cell. The importance of CBF in hematopoietic cells makes it a strong candidate for the factor that contributes to the leukemogenic properties of MLVs in vivo. However, CBF is not the only transcription factor that binds the Moloney and SL3-3 MLV core sites in vitro. Activating protein 3 (AP-3), mammalian C-type retrovirus enhancer factor 1 (MCREF-1), and SL3 core-binding factor (S-CBF) also bind MLV core sites. CBF is one of many proteins that bind MLV enhancers and one of several transcription factors that bind the core site. Here we show that the affinity of CBF for its DNA-binding site in Moloney MLV correlates with the latent period of disease onset and disease specificity of the virus. Altered Moloney viruses that contain high-affinity CBF binding sites induce predominantly thymic lymphoblastic lymphomas, whereas viruses with lower-affinity CBF binding sites lose the exquisite T-cell disease specificity of Moloney MLV. The results suggest that CBF, which was identified as a candidate host cell transcription factor responsible for the T-cell disease specificity of Moloney MLV (Jahner \& Jaenisch, 1985), may in fact be contributing to the Tcell disease specificity of this virus in vivo. It is also formally possible that it has inadvertently created high-affinity binding sites for proteins expressed in hematopoietic cells other than $T$ cells upon introducing low-affinity CBF binding sites into the Moloney MLV enhancer and that this caused alterations in disease specificity. The expression pattern of Cbfa2, which encodes the CBFa subunit originally purified from thymus tissue, may shed some light on why the lowest-affinity core sites in the Moloney MLV enhancer [SS5 and Mo(core)] tend to shift disease specificity toward erythro-leukemia. Cbfa2 is highly expressed in c-kit1 CD341 fetal liver hematopoietic stem cells and in bone marrow. Expression remains high in the thymus and in some myeloid lineages but appears to be rapidly reduced in both the primitive 
and definitive erythroid lineages (Loh et al., 1990; Hoffman et al., 1982). Thus, CBF may not be significant for Moloney MLV replication in cells of the erythroid lineage. Low-affinity core sites may selectively impair transcription of the Moloney MLV genome in lymphocytes and in myeloid lineages, but not in erythroid progenitors, hence shifting the distribution of leukemias towards the erythroid lineage.

\section{DNA METHYLATION AND LEUKEMIA VIRUS EXPRESSION}

Recent studies have shown evidence that methylation does not intervene to silence genes that are actively transcribed, but only affects genes that have already been shut down by other means. There is reason to believe that transcriptional activity may somehow imprint the methylation-free status of $\mathrm{CpG}$ islands. The involvement of DNA methylation in inactivation of transposable elements could likewise be due to its capacity for stabilizing the transcriptional shutdown organized by other systems (Bird, 2002).

Retroviral vectors based on the exogenous retrovirus (XRV) Moloney murine leukemia virus (MLV), such as the MFG vector (Walsh et al., 1998), and have been used extensively in the laboratory and in the clinic as vehicles for gene delivery. In both settings, transcriptional silencing has proven to be a major impediment to stable/long-term proviral expression (Natalie et al., 1977; Dodge et al., 2002), due in part to the presence of negative regulatory elements in the proviral LTR that are bound by transcriptional repressors (Leunga et al., 2011; Ellis, 2005) and the binding of chromatin proteins, including histone $\mathrm{H} 1$ (Harris \& Liddament, 2004) and macroH2A1 (Bestor, 2000). MLV is repressed with particular efficiency in embryonic carcinoma (EC) and embryonic stem (ES) cells (Flanagan et al., 1989). DNA methyltransferases, Dnmt3a and Dnmt3b, are required for de novo methylation in embryonic stem (ES) cells and postimplantation embryos. However, the mechanism of de novo methylation is largely unknown. In this study, we have analyzed the sequence specificity of Dnmt3a and Dnmt3b during de novo methylation of murine Moloney leukemia virus provirus DNA in virus-infected ES cells. Provirus DNA from infected wild-type (J1), Dnmt1-/- (c/c), and Dnmt3a3b-/- (3a3b-/-) ES cells were analyzed using the bisulfite sequencing method. It has been demonstrated that Dnmt3 enzymes methylate predominantly CpG sites in vivo and confirm that Dnmt3 enzymes, but not Dnmt1, are responsible for de novo methylation. However, the sequence context and $\mathrm{CpG}$ density do not appear to influence de novo methylation, though strand bias is detectable. Interestingly, non-CpG methylation is detected as a component of de novo methylation. MLV-based retroviral vectors similar to those used in this study have been used extensively for gene transfer studies and to deliver genes for therapeutic purposes (Tsukiyama et al., 1989). Despite the fact that MLV preferentially integrates within or near the promoter regions of genes (Pannell et al., 2000), DNA methylation and transcriptional silencing of such vectors are frequently observed (Yao et al., 2004), explaining in part why they have shown limited efficacy. The results presented here indicate that G9a plays an important role in the silencing of such vectors in endothelial stem cells and raises the question of whether G9a plays a similar role in other cell types.

\section{SUMMARY AND CONCLUSION}

It can be summarized that the expression and function of core-binding factors throughout hematopoietic development and in disease state as shown in Figure 1, are struck by the observation that almost all hematopoietic lineages save erythrocytes (both primitive and 
definitive) which seem to be affected at one or more points in their differentiation by mutations in core-binding factor genes.
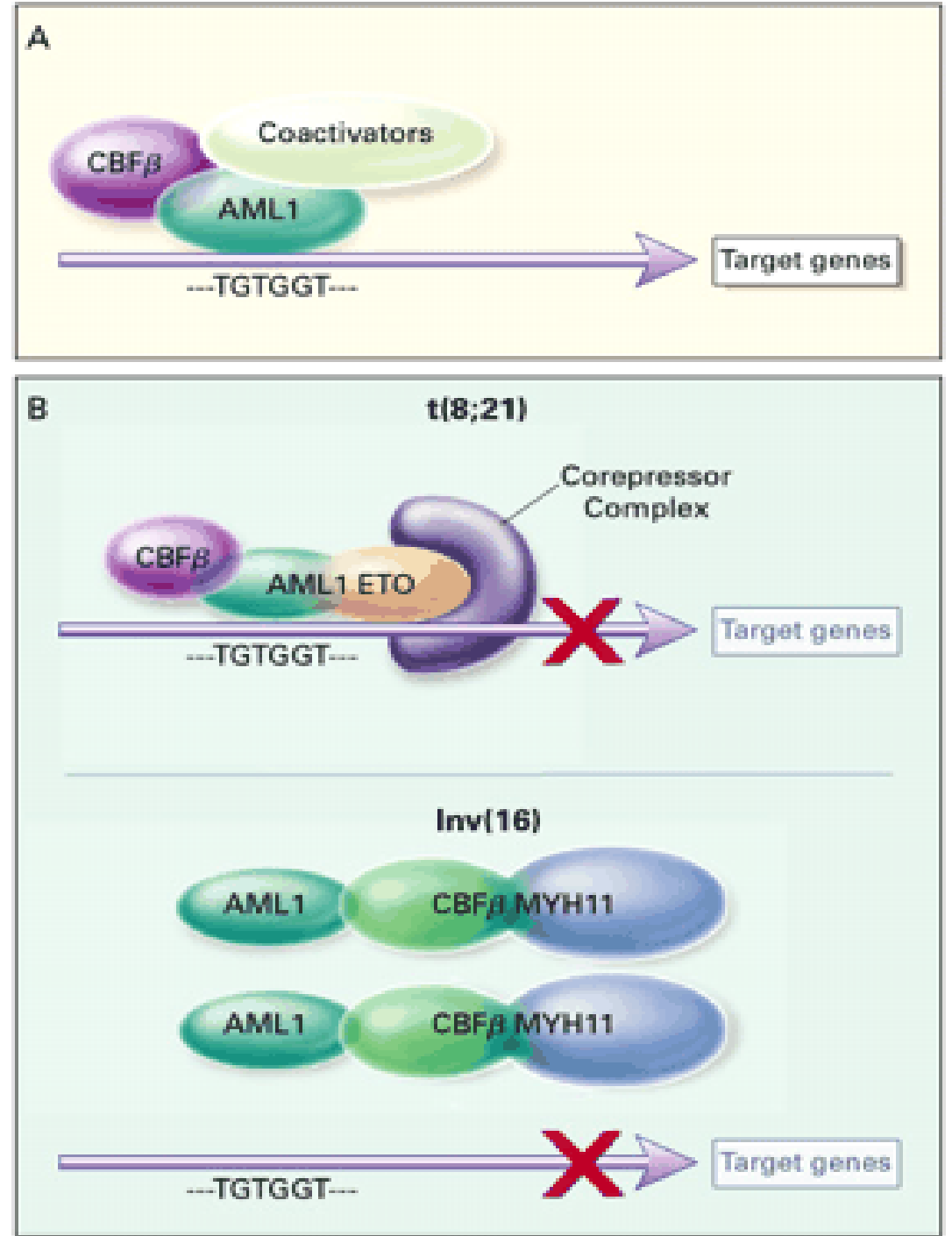

Fig. 1. The influence of CBF beta in progression of acute myeloid leukemia

These functions and expression patterns throw light on one of the original observations about core-binding factors and their role in murine retro-viral pathogenesis. One of several lines of investigation that led to the discovery of the core-binding factors was that they bound to the 'core' sites in the enhancers of retroviruses that caused T-cell leukemias, and a core binding activity was found to be particularly abundant in stem cells (Changolkar et al., 2008). A mutation in the core site of the Moloney murine leukemia virus changed its disease specificity from T-cell leukemia to erythroleukemia, and led to the hypothesis that the mutation disrupted the binding of a protein that is highly expressed in T cells, and caused transcription and replication of the virus to be selectively dampened in stem cells relative to 
erythroid progenitors (Jahner et al., 1982; de Bruijn and Speck, 2004). Thus epigenetic factors like DNA methylation and core binding factor are critical for expression of leukemia virus in stem cells and may contribute immensely to the understanding of erythroid dyscrasias.

\section{ACKNOWLEDGEMENTS}

The authors are thankful to Dr.Nancy Speck, Philadelphia, USA and the Biotechnology Department, AIMST University, Malaysia for help in the preparation of this manuscript.

\section{REFERENCES}

Bestor, T.H. (2000). Gene silencing as a threat to the success of gene therapy. J. Clin. Invest., 105, 409-411.

Bird, A. (2002). DNA methylation patterns and epigenetic memory. Genes Dev., 16, 6-21.

Cedar, H. (1998). DNA methylation and gene activity. Cell, 53, 3-4.

Changolkar, L.N., Singh, G., Pehrson, J.R. (2008). MacroH2A1-dependent silencing of endogenous murine leukemia viruses. Mol. Cell Biol., 28, 2059-2065.

Dixie, L., Magerb, Fabio Rossid, Matthew, C. Lorincz. (2011). Lysine methyltransferase G9a is required for de novo DNA methylation and the establishment, but not the maintenance, of proviral silencing. Proc. Natl. Acad. Sci., USA, 10(1073), 1-6.

Dodge, J.E., Ramsahoye, B.H., Wo, Z.G., Okano, M., Li, E. (2002). De novo methylation of MMLV provirus in embryonic stem cells: $\mathrm{CpG}$ versus non-CpG methylation. Gene., 289(1-2), 41-8.

Dranoff, G., et al. (1993). Vaccination with irradiated tumor cells engineered to secretemurine granulocyte-macrophage colony-stimulating factor stimulates potent, specific, and long-lasting anti-tumor immunity. Proc. Natl. Acad. Sci., USA, 90, 35393543

Ellis, J. (2005) Silencing and variegation of gamma retrovirus and lentivirus vectors.Hum Gene Ther., 16, 1241-1246.

Flanagan, J.R., Krieg, A.M., Max, E.E., Khan, A.S. (1989). Negative control region at the $5^{\prime}$ end of murine leukemia virus long terminal repeats. Mol. Cell Biol., 9, 739-746

Ganstch, J.W. (1980). Embryonal carcinoma stem cells lack a function required for virus replication. Nature, 285, 110-112

Harris, R.S., Liddament, M.T. (2004). Retroviral restriction by APOBEC proteins. Nat. Rev. Immunol., 4, 868-877.

Hoeben, R.C., Migchietsen, A.A., van der Jagt, R.C., van Ormundt, H., van der EB, A.J. (1991) Inactivation of the Moloney murine leukemia virus long terminal repeat in murine fibroblast cell lines is associated with methylation and dependent on its chromosomal position. J. Virol., 65(2), 904-912

Ivanov, A.A. (2011). Isotopic self programming for the embryonic Cell DNA methylation pattern. International Res. J. Pure Applied Chem., 1(2), 58-64

Jähner, D., et al. (1982) De novo methylation and expression of retroviral genomes during mouse embryogenesis. Nature, 298, 623-628.

Jahner, D., Jaenisch, R. (1985). Retrovirus induced de novo methylation of flanking host sequences correlates with gene in activity. Nature, 315, 594-597. 
Joseph, Hoffman, Steffen, D., Gusella, J., Tabin, C., Bird, S., Cowing, D., Weinberg, R.A. (1982). DNA Methylation Affecting the Expression of Murine Leukemia Proviruses. J. Virology, 44(1), 144-157.

Kundu, M., Chen, A., Anderson, S., Kirby, M., Xu, L., Castilla, L., Bodine, D.M., Lui, P.P. (2002). Role of $\mathrm{Cbfb}$ in hematopoiesis and perturbations resulting from expression of the leukemogenic fusion gene Cbfb-MYH11. Blood, 100, 2449-2456.

Leunga, Danny C., Donga, Kevin B., Maksakovaa, Irina A., et al. (2011). Lysine methyltransferase G9a is required for de novo DNA methylation and the establishment, but not the maintenance, of proviral silencing. Proc. Natl. Acad. Sci., USA, www.pnas.org/cgi/doi/10.1073/pnas.1014660108.

Loh, T.P., Sievert, L.L., Scott, R.W. (1990). Evidence for a stem cell specific repressor of Moloney murine leukemia virus expression in embryonal carcinoma cells. Mol. Cell. Biol., 10, 4045-4057.

Marella, F.T.R., de Bruijn, Nancy, A. Speck. (2004). Core-binding factors in hematopoiesis and immune function. Oncogene, 23, 4238-4248.

Natalie, M. Teich, Robin, A. Weiss. (1977). Virus infection of murine teratocarcinoma stem cell lines. Cell, 12(4), 973-98.

Niki, M., Okada, H., Takano, H., Kuno, J., Tani, K., Hibino, H., Asano, S., Ito, Y., Satake, M., Noda, T. (1997). Hematopoiesis in the fetal liver is impaired by targeted mutagenesis of a gene encoding a non-DNA binding subunit of the transcription factor, polyomavirus enhancer binding protein $2 /$ core binding factor. Proc. Natl. Acad. Sci., USA, 94, 5697-5702.

Pannell, D., et al. (2000) Retrovirus vector silencing is de novo methylase- independent and marked by a repressive histone code. Embo. J., 19, 5884-5894.

Sasaki, K., Yagi, H., Bronson, R.T., Tominaga, K., Matsunashi, T., Deguchi, K., Tani, Y., Kishimoto, T., Komori, T. (1996). Absence of fetal liver hematopoiesis in mice deficient in transcriptional coactivator core binding factor beta. Proc. Natl. Acad. Sci., USA, 93, 12359-12363.

Tsukiyama, T., Niwa, O., Yokoro, K. (1989). Mechanism of suppression of the long terminal repeat of Moloney leukemia virus in mouse embryonal carcinoma cells. Mol. Cell Biol., 9, 4670-4676.

Walsh, C.P., Chaillet, J.R., Bestor, T.H. (1998). Transcription of IAP endogenous retro viruses is constrained by cytosine methylation. Nat. Gene., 20, 116-117

Wang, Q., Stacy, T., Miller, J.D., Lewis, A.F., Huang, X., Bories, J.C., Bushweller, J.H., Alt, F.W., Binder, M., Marıon-Padilla, M., Sharpe, A., Speck, N.A. (1996). Cell, 87, 697708.

Yao, S., et al. (2004). Retrovirus silencing, variegation, extinction, and memory are controlled by a dynamic interplay of multiple epigenetic modifications. Mol. Ther., 10, 27-36.

Yoder, J.A., Walsh, C.P., Bestor, T.H. (1997). Cytosine methylation and ecology of intragenomic parasites. Trends Genet., 13, 335-340.

(c) 2011 Tyagi \& Kumar; This is an Open Access article distributed under the terms of the Creative Commons Attribution License (http://creativecommons.org/licenses/by/3.0), which permits unrestricted use, distribution, and reproduction in any medium, provided the original work is properly cited. 